\title{
PEDIATRIC PYOGENIC SACROILIITIS: A RARE CASE REPORT
}

\author{
Bipan Shrestha ${ }^{1}$ Kishor Man Shrestha ${ }^{1}$ Alok Pandey ${ }^{2}$
}

\section{ABSTRACT}

\section{INTRODUCTION}

Pyogenic sacroiliitis accounts for $12 \%$ of all cases of septic arthritis with less than 200 cases reported in the English literature since the beginning of the twentieth century. Considerable delay between presentation and diagnosis is seen because of unusual presentation and diagnostic dilemma. Cultures of joint fluid usually grow Staphylococcus aureus. Magnetic resonance imaging has been found to be the most useful imaging modality in diagnosis. Being very uncommon disease in children, the key to successful management is early diagnosis in which computed tomography (CT), bone scans, and magnetic resonance imaging (MRI) findings play a crucial role. If the diagnosis is established promptly, most patients can be managed successfully with antimicrobial therapy with excellent prognosis. Most reported cases required prolonged antimicrobial therapy of six to nine weeks. Presented here is a child with pyogenic sacroiliitis managed at our hospital (Universal College of Medical Sciences Teaching Hospital-UCMSTH) and review of the literature on this relatively rare diagnosis.

KEYWORDS Pyogenic, sacroiliitis, Staphylococcus, paediatric, rare disease

1. Lecturer, Dept. of Orthopaedics \& Trauma Surgery, Universal College of Medical Sciences, Bhairahawa, Nepal

2. Associate Professor, Dept. of Orthopaedics \& Trauma Surgery, Universal College of Medical Sciences, Bhairahawa, Nepal

\author{
For Correspondence \\ Dr. Bipan Shrestha \\ Department of Orthopaedics \& Trauma Surgery \\ Universal College of Medical Sciences \\ Bhairahawa, Nepal \\ Email: bipansht@hotmail.com
}




\section{INTRODUCTION}

Pyogenic sacroiliitis is relatively a rare condition, representing only $1-2 \%$ of all cases of septic arthritis in children. Initial symptoms are usually nonspecific and difficult to differentiate from septic arthritis of the hip. Diagnosis of pyogenic sacroiliitis has been difficult in the past due to its deep location and may be delayed due to the lack of specific clinical signs. Delay in diagnosis may lead to several complications, such as abscess or sequestration formation, prolonged period of sepsis, and long-term joint deformity. Newer diagnostic techniques such as bone scanning, computed tomography (CT), and magnetic resonance imaging (MRI) aid in early diagnosis and treatment. We present a case of pyogenic sacroiliitis in 10 year old child.

\section{CASE REPORT}

A 10-year-old girl presented with a 7 day history of acute onset, non-radiating, right posterolateral hip and gluteal region pain. The pain was initially accompanied by local swelling without erythema and progressed to painful ambulation. There was no history of nausea, vomiting, diarrhea, sore throat, skin infection or rash; however, there was one day history of fever but no chills and rigors. Past medical, surgical, social, and familial history was noncontributory. Vital signs were notable for a temperature of 100 degrees Fahrenheit (F) and heart rate of 120 beats per minute (bpm) at the time of admission. Physical examination was notable for severe tenderness at the right gluteal region and right hip joint with painful passive and active flexion, extension, internal and external rotation of right hip joint. Flexion abduction and external rotation (FABER) test was positive for right sacroiliac joint. Neuromuscular function was intact and the remainder of the physical exam was unremarkable. Laboratory studies demonstrated a white blood cell (WBC) count of 11,700 cells per cubic millimeter with $80 \%$ neutrophils, positive anti-streptolysin O (ASO) antibody, erythrocyte sedimentation rate (ESR) of 55 millimeters per hour, positive $\mathrm{C}$-reactive protein (CRP), normal renal function test and negative serological test for HIV and HBsAg. X-ray of the pelvis with both hip and sacroiliac joint was normal (Fig 1). Ultrasonography (USG) reported features suggestive of fat necrosis in subcutaneous plane of right gluteal region. Computed tomography with oral and intravenous contrast of the abdomen, pelvis, and hips was negative. The patient was admitted for further evaluation and begun on intravenous ceftriaxone and tazobactum combination, analgesics and rest in skin traction. Blood culture sent was negative for any organism growth. Finally MRI of the lumbar spine, pelvis, hip and Sacro-iliac joint demonstrated increased joint fluid in the right sacroiliac joint with bone marrow edema in the adjacent right sacrum with increased signal intensity and, confirming a diagnosis of septic sacroiliitis (Fig 2). After going through various literatures, we believed that surgical debridement was not necessary in this case and planned to continue medical treatment. The patient was then switched on to intravenous (IV) flucloxacillin and ofloxacin after the MRI report with the diagnosis of pyogenic sacroilitis. Traction was continued for ten days and patient improved clinically. Then patient was allowed to bear weight and walk. At the time of discharge she could walk with minimal pain with marked improvement in her symptoms. After 14 days of IV antibiotics patient was switched to same oral antibiotics for one more month and was discharged on it. At day 7 and day 14, her total count was 11400 and 11300 , Neutrophil $70 \%$ and $65 \%$ and ESR was 50 and 30, CRP positive and negative respectively.

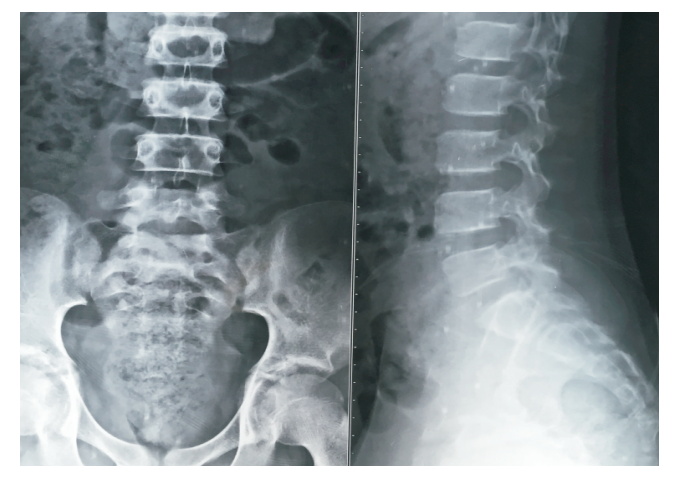

Fig 1. Xray of LS spine with bilateral SI joint showing normal finding

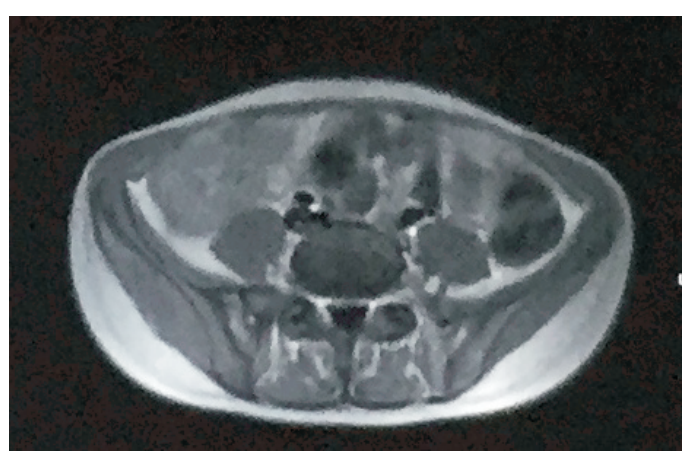

Fig 2. MRI pelvis with bilateral SI joint showing collection in right SI joint with edematous changes over sacrum and iliac bone juxtra-articular to SI joint

At 6 weeks follow-up, patient had occasional pain only after a long walk which later subsided at 3 month follow up. The erythrocyte sedimentation rate at $6^{\text {th }}$ week was 15 millimeters per hour. A repeat magnetic resonance imaging (MRI) and computed tomography (CT) showed no evidence of infection and sequestrum formation. 


\section{DISCUSSION}

The evaluation of a child with a limp involves consideration of several potential etiologies including infection, fracture, and rheumatic arthritis ${ }^{1}$. Septic sacroiliitis, also known as pyogenic sacroiliitis (PS), is an uncommon diagnosis in the pediatric patient, accounting for one to two \% of all cases of septic arthritis ${ }^{2}$, with fewer than 200 cases of PS having been reported in the English literature ${ }^{3}$ since the beginning of the twentieth century.

Initial symptoms are usually nonspecific and difficult to differentiate from septic arthritis of the hip. Diagnosis of pyogenic sacroiliitis has been difficult in the past due to its deep location and may be delayed due to the lack of specific clinical signs. Delay in diagnosis may lead to several complications, such as abscess or sequestration formation, prolonged period of sepsis, and long-term joint deformity ${ }^{4}$. Newer diagnostic techniques such as bone scanning, computed tomography $(\mathrm{CT})$, and magnetic resonance imaging (MRI) aid in early diagnosis and treatment ${ }^{5}$.

Schaad et al. ${ }^{6}$ reported 77 patients with sacroiliitis between 1961 and 1980 noting that infection was more common in boys during late childhood and in boys often presenting subacutely. Wu et al. reported a series of 33 cases of pyogenic sacroiliitis and they identified 11 cases aged less than 15 years ${ }^{7}$. Wada et al. reviewed eight pediatric patients with sacroiliitis identified between 2000 and $2005^{8}$. Recently Molinos Quintana et al reported 11 patients aged less than 14 years who met the criteria of pyogenic sacroiliitis during eight years (20022010) ${ }^{9}$. Bone scans were generally helpful in making the diagnosis, and aspiration of the joints frequently revealed Staphylococcus aureus as the infecting agent. Management frequently required antibiotic targeted towards Staphylococcus aureus and surgical debridement or drainage and the prognosis was excellent ${ }^{9}$.

Since 1980, there have been six case series which included five or more children ( $<18$ years old) that include outcomes. Staphylococcus aureus was the most frequently recovered pathogen however, case reports have also identified Salmonella $^{10}$ and Brucella ${ }^{11,12}$ as less frequent organisms. Previously there were a total number of 39 patients in the six case series ${ }^{6-10,13}$ most of whom were older than 10 years, although the youngest reported child was 20 months old. Patients group at increased risk of pyogenic sacroiliitis includes children with immuno suppressed state and children with sickle cell disease ${ }^{14}$. Trauma is an important predisposing factor with an estimated $10 \%$ of cases having a prior history of pelvic trauma ${ }^{15}$. Other predisposing factors include atopic dermatitis, insect bite, folliculitis and furunculosis ${ }^{9}$. However our patient had no preceding infection or traumatic injury. Our patient presented with acute course. As with our patient, signs and symptoms were relatively nonspecific, and patient was often admitted with alternative diagnoses. The physical examination was often nonspecific, with difficulty in localizing pain to the sacro-iliac (SI) joint. Aprin and Turen ${ }^{13}$ noted that 7 of 7 patients were positive in the FABER test. Similarly, Ncube ${ }^{16}$ noted that five of six patients had a positive sacroiliac stress test. The differential diagnosis is broad including appendicitis, tumours, discitis, and septic arthritis of the hip or clinical sepsis ${ }^{17}$. Due to nonspecific clinical picture in most of the cases there is a considerable delay between presentation and diagnosis 9 . Most cases of pyogenic sacroiliitis are unilateral, right sacroiliac joint is more frequently involved than left side for unknown reason; bilateral involvement also is not uncommon ${ }^{7}$.

There is no specific blood test which points to the diagnosis of pyogenic sacroiliitis; WBC count may be increased or normal; ESR and CRP may be elevated in the majority of cases, but while they are sensitive, they may not be specific ${ }^{18}$. Blood cultures should be performed before antibiotic therapy. The overall positive blood culture rate is low in pediatric patients $(45.5 \%)^{7}$. Nevertheless, local synovial fluid cultures have a high yield rate for pathogens. Sacroiliac joint synovial fluid aspiration is technically difficult due to the joint being deep seated and oblique and thus relatively inaccessible ${ }^{17}$; however this invasive procedure is not warranted routinely ${ }^{7}$; it is recommended in patients with clinical and radiographic features suggestive of pyogenic sacroiliitis, but with negative blood cultures, poor responder or non responder to conventional antibiotic therapy unlike our case where patient was responding to antimicrobial theray ${ }^{9}$. Roentograms of the hips are generally non diagnostic in pyogenic sacroiliitis (3 of 30 including our case). CT scans were positive in 12 of 15 cases and bone scans in 28 of 35 cases. The study by Doita et $a l{ }^{18}$ is the only series to include MRI findings which were positive in 5 of 5 cases. The authors noted that both MRI and bone scan were sensitive for PS; however, bone scans may be negative if done too early after onset of symptoms. Indeed, Moyer et $a l .{ }^{19}$ noted one case where an equivocal bone scan became positive when repeated 30 days later and another where a negative bone scan became positive four days later. In addition, bone scan cannot differentiate PS from psoas or gluteal abscesses or identify infection of adjacent tissue; therefore, MRI may provide more diagnostic accuracy, achieving high sensitivity and specificity ${ }^{18}$.

Traditionally, extended-spectrum penicillins or first generation cephalosporins have been the antibiotic of choice. However, methicillin-resistant Staphylococcus aureus will require clindamycin, linezolid, or trimethoprimSulfamethoxazole. Most patients had excellent outcomes, although, residual asymptomatic roentogram changes were noted in 12 of 24 unlike our case where no changes seen in $\mathrm{x}$ ray. Abscesses were reported in 3 of 30 (including current case) patients. Though, there is little pediatric data with 
respect to surgical drainage, Hodgson ${ }^{20}$ reported a series of 12 adult patients, of whom 11 had surgical drainage. All had a good outcome, though several had mild residual symptoms and one developed a draining sinus. In the literature $30-40 \%$ mortality rate were also reported prior to the antibiotic era ${ }^{2}$ onservative management therapy has been proved to be effective in a series of patients with soft tissue abscesses ${ }^{8}$. However surgical drainage is indicated only in the presence of sequestrum formation, osteomyelitis, and failure of medical management ${ }^{17,21}$.

Pyogenic sacroiliitis should always be included in the differential diagnosis of any child with fever with buttock, hip, or back pain. FABER test should be performed routinely in these patients and if positive, an MRI is recommended to rule out pyogenic sacroiliitis. Antibiotic therapy has proved effective in most cases with good clinical response and with no sequelae during followup.

\section{CONCLUSION}

Pyogenic sacroiliitis is quite a rare disease in children and remains a diagnostic challenge. Signs and symptoms can be nonspecific, and physical examination is often unhelpful. The most important clinical feature is gluteal and hip pain, fever and limp. The FABER test is thought to be useful in evaluating the sacroiliac joint. When inflammatory parameters are elevated they are consistent with the diagnosis, but their absence is not reassuring. Blood cultures and aspiration of the SI joint are useful in identifying the infecting agent. While imaging may include roentogram, bone scan, CT, and MRI with varying degrees of sensitivities, MRI may be the most sensitive and specific out of these in securing the correct diagnosis. Antibiotic therapy initially should be started empirically towards Staphylococcus aureus and then continued for at least three weeks. The optimum duration of therapy is not known, though in most cases antibiotics were continued for six to nine weeks. Consistent with patient previously described in other reports our patient ultimately responded to antibiotic therapy with good final outcomes.

\section{REFERENCES}

1. Tse SM, Laxer RM. Approach to acute limb pain in childhood. Pediatr Rev. 2006;27:1709.

2. Attarian DE. Septic sacroitiitis: the overlooked diagnosis. J South Orthop Assoc. 2001;10:5760.

3. Ford LS, Ellis AM, Allen HW, Campbell DE. Osteomyelitis and pyogenic sacroiliitis: A difficult diagnosis. J Paediatr Child Health. 2004;40:3179.

4. U. B. Schaad, G. H. McCracken, and J. D. Nelson, "Pyogenic arthritis of the sacroiliac joint in pediatric patients," Pediatrics. 1980;66(3):375379
5. G. T. Abbott and H. Carty, "Pyogenic sacroiliitis, the missed diagnosis?"British Journal of Radiology. 1993;66(782) : 120122 .

6. Sahaad UB, McCracken GH, Jr., Nelson JD. Pyogenic arthritis of the sacroiliac joint in pediatric patients.Pediatr. 1980;66:3759.

7. M. S. Wu, S. S. Chang, S. H. Lee, and C. C. Lee, "Pyogenic sacroiliitisa comparison between paediatric and adult patients,"Rheumatology. 2007;46(11):16841687

8. A. Wada, K. Takamura, T. Fujii, H. Yanagida, and P. Surijamorn, "Septic sacroiliitis in children," Journal of Pediatric Orthopaedics. 2008;28(4):488492.

9. A. Molinos Quintana, B. Morillo Gutiérrez, M. S. Camacho Lovillo, O. Neth, and I. Obando Santaella, "Pyogenic sacroiliitis in childrena diagnostic challenge," Clinica Rheumatology. 2011;30(1):107113.

10. Feldman LS. Salmonella septic sacroiliitis: case report and review. Pediatr Infect Dis J. 2006;25:1879.

11. Ariza J, Pujol M, Valverde J, et al. Brucellar sacroiliitis: findings in 63 episodes and current relevance. Clin Infect Dis. 1993;16:7615.

12. Pourbagher A, Pourbagher MA, Savas L, et al. Epidemiologic, clinical, and imaging findings in brucellosis patients with osteoarticular involvement. Am J Roentgenol. 2006;187:87380.

13. Aprin H, Turen C. Pyogenic sacroiliitis in children. Clinl Orthop Relat Res. 1993:98106.

14. A. A. Osman and S. Govender, "Septic sacroiliitis," Clinical Orthopaedics and Related Research. 1995;313:214219.

15 J. J. Vyskocil, M. A. McIlroy, T. A. Brennan, and F. M. Wilson, "Pyogenic infection of the sacroiliac joint. Case reports and review of the literature,"Medicine. 1991;70(3):188197.

16. Ncube BA. Pyogenic sacroiliitis in children. Br J Clin Pract. 1988;42:1547.

17. R. Raman, H. Dinopoulos, and P. V. Giannoudis, "Management of pyogenic sacroilitis: an update,"Current Orthopaedics. 2004;18(4):321325.

18. Doita M, Yoshiya S, Nabeshima Y, et al. Acute pyogenic sacroiliitis without predisposing conditions.Spine.2003;28 E3849.

19. Moyer RA, Bross JE, Harrington TM. Pyogenic sacroiliitis in a rural population. J Rheumatol.1990;17:13648.

20. Hodgson BF. Pyogenic sacroiliac joint infection. Clin Orthop Relat Res. 1989:1469.

21. M. Grippi, L. E. Zionts, E. R. Ahlmann, D. M. Forrester, and M. J. Patzakis, "The early diagnosis of sacroiliac joint infections in children," Journal of Pediatric Orthopaedics.2006;26(5) :589593. 